\title{
Research on Temperature Control Technology of Thermal Vacuum Test in Aerospace Field
}

\author{
Juan Ning ${ }^{1}$ a, Jing-yi Shao ${ }^{1}$, Ying Zhou ${ }^{1}$ and Yang-yang Liu ${ }^{1}$. \\ ${ }^{1}$ Beijing Institute of Satellite Environment Engineering, Beijing, 100094, P.R.China
}

\begin{abstract}
The temperature control system is an indispensable key system for the thermal vacuum test. It is used to simulate the infrared heat flow environment of the spacecraft in orbit. In order to improve the stability and reliability of temperature control, this paper designs and develops a thermal vacuum test temperature control system based on the Modbus RTU serial communication protocol on the LabVIEW platform. The system combines the flexibility of software development on LabVIEW platform and combines the stability of serial communication with controller. This temperature control system has been used in thermal vacuum test, the control effect of overshooting within $1{ }^{\circ} \mathrm{C}$ and temperature control accuracy within $\pm 0.2^{\circ} \mathrm{C}$. The system has high stability, which achieves the expected goal.
\end{abstract}

\section{Introduction}

The thermal vacuum test equipment can be used to simulate high and low temperature space environment, and has a wide range of applications in the aerospace field. The quality of its environmental test directly affects the evaluation of satellite quality. Therefore, the environmental test process should be accurately controlled ${ }^{[1]}$. The temperature control system is an indispensable key system for the thermal vacuum test. It is used to simulate the infrared heat flow environment of the spacecraft in orbit. The data provides calculation and correction basis for the heat flow analysis model such as spacecraft and specimen. The thermal control system of the thermal vacuum test equipment mainly has the following technical difficulties: (1) The temperature response is delayed due to the influence of environment and equipment; (2) The thermal model is not uniform due to the diversity of specimen, and the control parameters cannot be predicted; (3) Sudden change in thermal load of the specimen itself causes temperature overshoot; (4) The test has strict requirements for overshoot and the linearity of temperature curve ${ }^{[2]}$. Aiming at the characteristics of thermal vacuum test, a thermal vacuum test temperature control system is designed and developed based on Modbus RTU serial communication protocol on the LabVIEW platform. The system is based on VISA's programming mechanism to realize serial communication between LabVIEW and temperature control instrument. VISA supports many types of interfaces, powerful functions and mature technology. It is one of the most widely used tools for virtual instrument and control programming ${ }^{[3]}$.

\section{The composition of the temperature control system for thermal vacuum test}

The thermal vacuum test equipment is basically composed of a chamber, a heat sink system, a refrigeration system, a vacuum system, and a measurement and control system. In the refrigeration mode, it can be generally divided into liquid nitrogen refrigeration, refrigerant refrigeration, gas nitrogen temperature regulation, bath oil temperature regulation, etc. The heating method also has an infrared heating cage to adjust the temperature. The thermal vacuum test temperature control system is mainly composed of sensors, temperature controller, program-controlled power supply, heating device and measurement and control computer. The schematic diagram of the system is shown in Figure 1. The software is based on the Windows system platform, LabVIEW high-level language programming, applying modular programming ideas, and dividing the system functions into different modules. The main program calls different function modules through mode selection. The output of the SR23 temperature controller is controlled by an analog quantity, thereby adjusting the output power of the programmable power supply to adjust the temperature of the heating device. The temperature value of the temperature-controlled object measured by the platinum resistance PT100 is fed back to the measurement and control software is designed on LabVIEW platform to complete the temperature control process ${ }^{[4]}$.

${ }^{a}$ Corresponding author: ningjuan_buaa_ee@126.com 


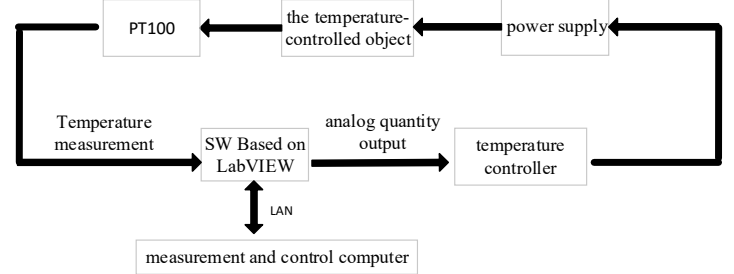

Figure1. Temperature control system schematic

\section{Software design and development of temperature control system for thermal vacuum test}

The temperature control system software functions are mainly divided into two parts: function setting content communication and parameter reading content communication. The function setting contents include: remote COM port setting, temperature controller standby setting, auto-tuning function setting, setting pre-control temperature value, etc. The parameter reading function includes sensor feedback value and output power percentage, etc.

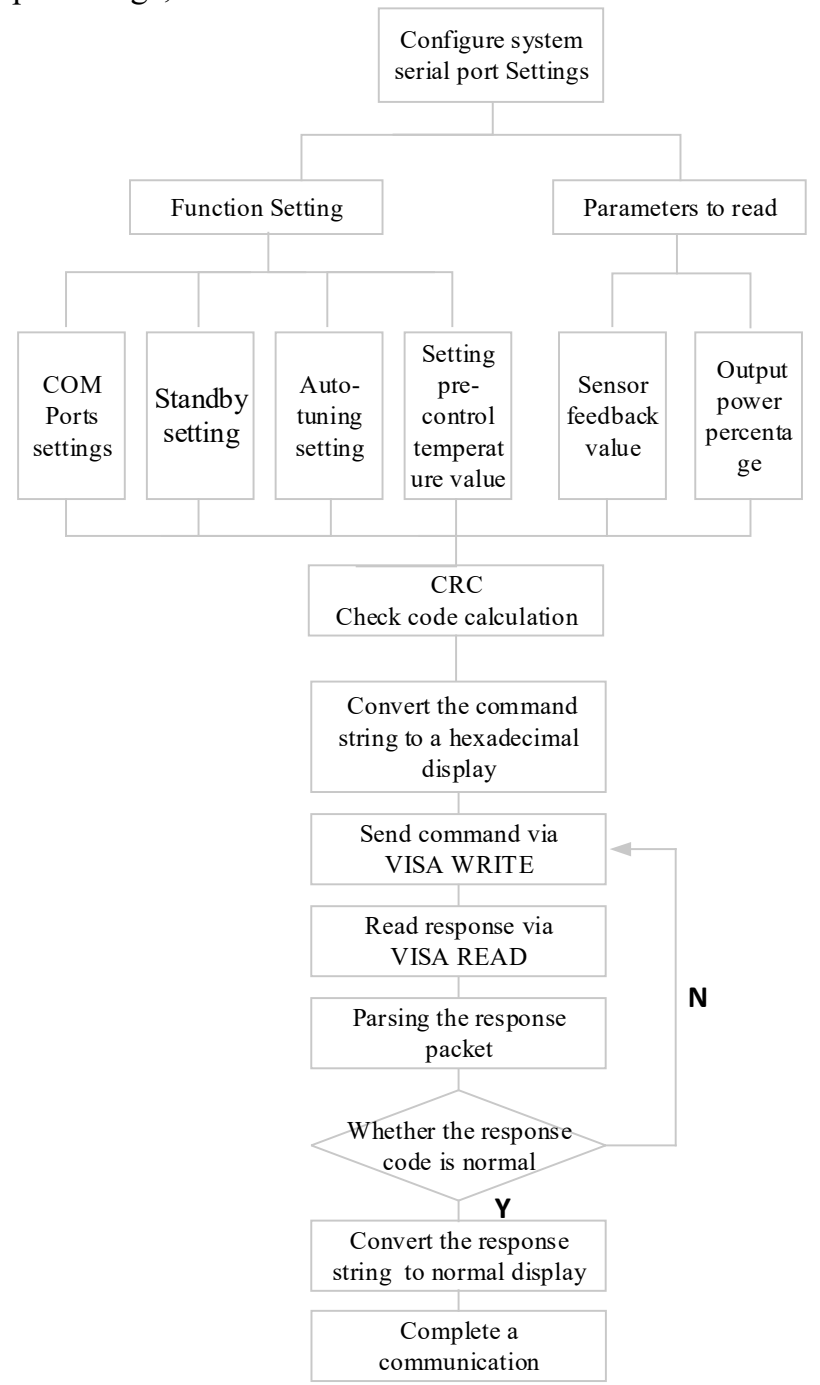

Figure2. The system function logic flow chart

First, the temperature control system performs system serial port configuration and initializes the specified serial port according to a specific setting. Then, the system sends an instruction to perform CRC cycle check code calculation. Convert the normal display of the command string to a hexadecimal display. Write the data to the hardware device through VISA WRITE, and read a specified number of bytes in the hardware device via VISA READ and return the data to the read buffer. Parsing the response packet to determine whether the response code is normal. If not, resend the command, and if normal, convert the response string from hexadecimal to normal display. The system function logic flow chart is shown as in Figure 2

\subsection{Modbus-RTU communication settings}

The communication between the LabVIEW platform and the SR23 temperature controller is based on the ModbusRTU protocol. The Modbus communication protocol is used as a general industry standard for communication between controllers or between controllers and other devices. The Modbus communication protocol has two message frame formats: ASCII and RTU (Remote Terminal Unit). The ASCII mode uses LRC check, the RTU mode uses CRC check, and the RTU mode is more suitable for the case of a large amount of transmitted data. The CRC check is much more complex than the LRC check ${ }^{[5]}$. The Modbus communication protocol uses the master-slave technology, that is, the master device performs initialization query transmission, and the slave responds based on the data provided by the master device. Here the master device is the host computer and the slave device are the SR23 temperature controller. Figure 3 below shows the program of the LabVIEW serial communication setup.

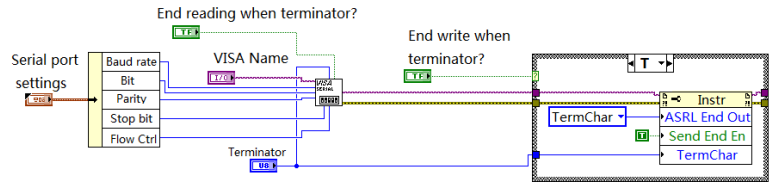

Figure3. Front program of LabVIEW serial communication Settings

\subsection{Cycle redundancy check}

The CRC Redundancy Check is shortened cyclic codes. It is widely used in frame check and is a data check code with strong error checking capability. The calculation method of CRC code is as follows: First, pre-set a 16-bit CRC register to hexadecimal FFFF (that is, the total for 1 ), and then XOR the first byte of the communication message frame with the lower 8 bits of the 16-bit CRC register, put the result in the $\mathrm{CRC}$ register, then shift the contents of the CRC register to the right by one bit (toward the lower bit), fill the highest bit with 0 , and check the shift bit after the right shift. If the shift bit is 1 , the CRC register is XORed with the polynomial A001. Repeat the above step, shift to the right 8 times, and the entire 8-bits of the first byte are processed. Repeat the above steps to process the next byte of the communication information frame. After all the bytes of the communication information frame are calculated according to the above steps, the high and low bytes of 
the 16-bit CRC register are exchanged to obtain the CRC code ${ }^{[6]}$. The specific implementation method is as shown in Figure 4.

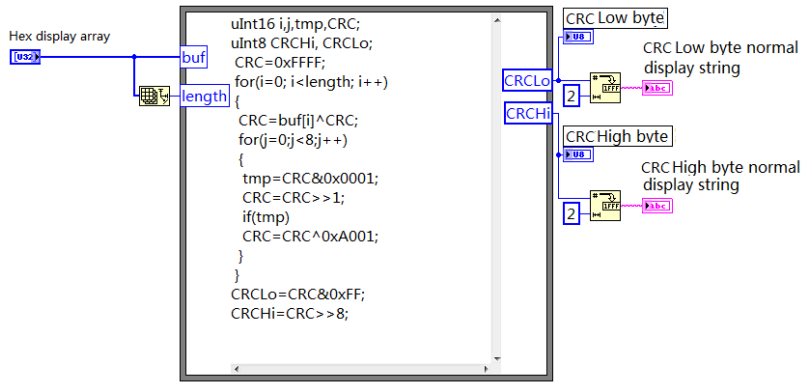

Figure4. CRC redundancy check code block program

\subsection{Function triggering and command optimization sending}

The temperature control program uses the event structure, its function settings include the following sections: event- 1 is the communication setting function, event- 2 is the self-tuning function, event- 3 is the standby function, and even- 4 is the pre-controlled temperature value setting function. The temperature control reading parameter includes the current measurement value and the output power percentage. After the program is started, the two parameter values are cyclically read. When the parameter setting of the temperature control function changes, the parameter reading function is suspended, and the parameter setting of the temperature control function is started. Through a Boolean variable to control the order in which read and control instructions are sent. Figure 5 below is the block program of the event-1 communication setting function. Event-2 and 3 are Boolean type events, which are consistent with the event-1 principle. Figure 6 below is the event- 4 precontrol temperature value setting block program, which belongs to the Value type event.

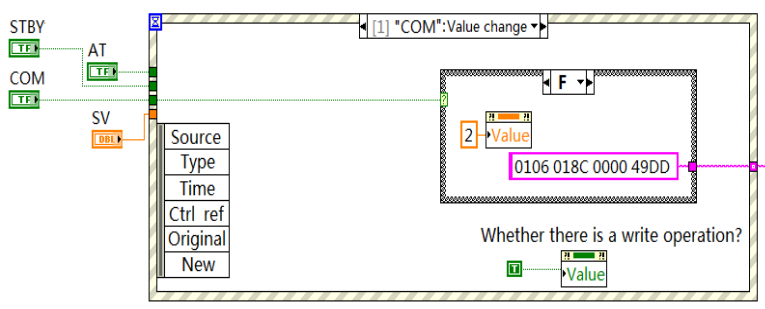

Figure5. Event-1 the communication setting function block

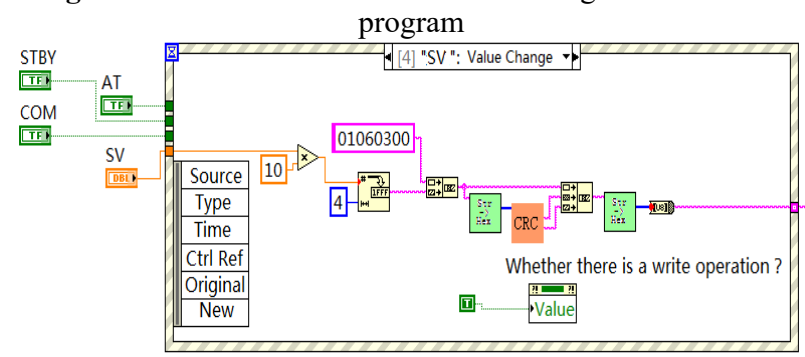

Figure6. Event-4 pre-control temperature value setting block program

After the measurement parameter read command is sent successfully, the temperature controller will return the measured temperature present value, the output power percentage and other parameters. The block program of the measurement parameter reading function is shown in Figure7 below. The software stores the data in the database TDMS (Technical Data Management Steaming) and stores it in binary data type. It has the advantages of fast reading and writing speed and large storage capacity, and adds real-time curve and report functions on the front panel to facilitate user operation ${ }^{[7]}$

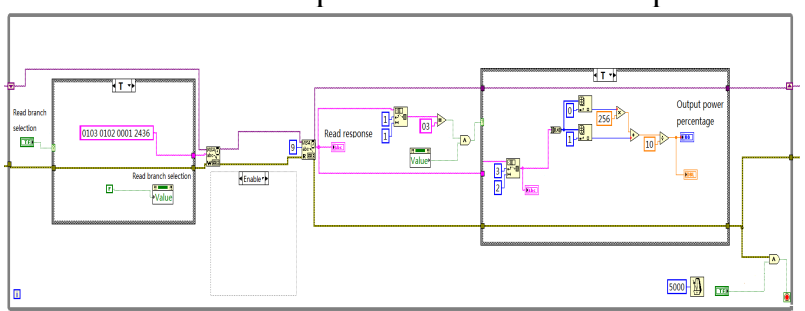

Figure7. The block program of the measurement parameter reading function

The temperature control parameter setting has corresponding execution controls on the front operation panel. Each control completes a temperature control function, so each control generates an event, and the generated event is relatively independent. Click the control on the operation panel to trigger the background program and enter the corresponding event. The temperature controller completes the event function through the serial port communication. A part of the program of the temperature control parameter setting function is shown in Figure 8.

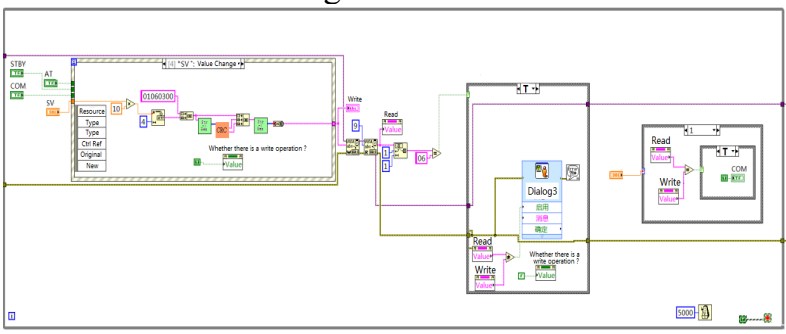

Figure8. A part of the program of the temperature control parameter setting function

The temperature control software operation interface of the thermal vacuum test equipment is shown in Figure 9. The temperature control software has real-time data display, real-time control, curve, report, storage and other functions. The main interface of the software has communication port settings, which can set communication parameters of the temperature control instrument, remotely control the temperature control instrument, monitor the temperature control instrument data, set the temperature control parameters, record mode and path, and can display the send command and command response. In the software interface, the temperature data and curve of the temperature control point can be displayed and stored in real time. At the end of each test, the stored data can be exported from the TDMS database, and the file name is defined by the user. 


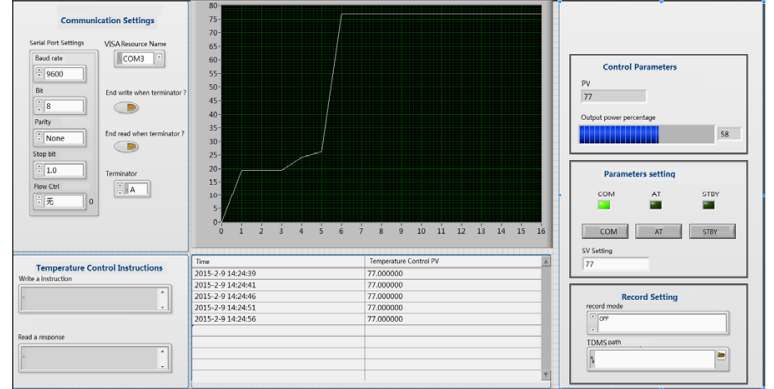

Figure9. Thermal vacuum test equipment temperature control software operation interface

\section{Thermal vacuum temperature control test results and analysis}

After the vacuum and heat sink temperature of the thermal vacuum test reach the technical specifications, the automatic temperature control program is run to carry out the high and low temperature alternating test. The test contains 2.5 cycles, and the specimen has an alternating range of $-70^{\circ} \mathrm{C}$ to $+90^{\circ} \mathrm{C}$. The test procedure is as follows:

a. The target temperature of the specimen is reset to $+90{ }^{\circ} \mathrm{C}$. After reaching the temperature, the high temperature is maintained for 2 hours.

b. The target temperature of the specimen is reset to $70{ }^{\circ} \mathrm{C}$. After reaching the temperature, the low temperature is maintained for 2 hours, and the first cycle is completed;

c. Repeat the above steps once to complete the second cycle;

d. After the completion of 2 cycles, set the temperature of the specimen to $+35^{\circ} \mathrm{C}$, and heat sink temperature rises.

The temperature control curve of the thermal vacuum test is shown in Figure 10 below. Figure 11 and Figure 12 show the temperature fluctuation error curves at $+90^{\circ} \mathrm{C}$ and $-70^{\circ} \mathrm{C}$ respectively. At a high temperature steady state of $+90^{\circ} \mathrm{C}$, the control temperature fluctuates between $+90.1{ }^{\circ} \mathrm{C}$ and $+89.9^{\circ} \mathrm{C}$, and the overshoot is $0.9^{\circ} \mathrm{C}$. At a low temperature steady state of $-70^{\circ} \mathrm{C}$, the control temperature fluctuates between $-70.2^{\circ} \mathrm{C}$ and $70{ }^{\circ} \mathrm{C}$, and the overshoot is $0.2^{\circ} \mathrm{C}$. The test system achieves the control effect of overshooting within $1{ }^{\circ} \mathrm{C}$ and temperature control accuracy within $\pm 0.2^{\circ} \mathrm{C}$, achieving the desired goal.

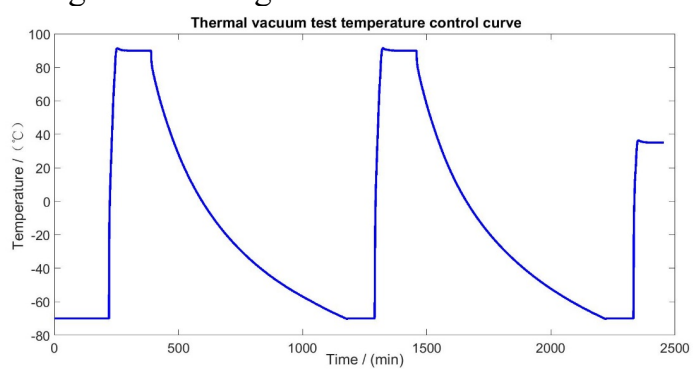

Figure10. Thermal vacuum test temperature control curve

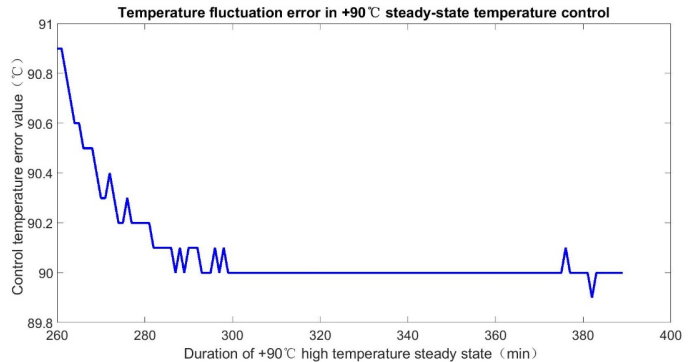

Figure11. Temperature fluctuation error in $+90^{\circ} \mathrm{C}$ steady-state temperature control

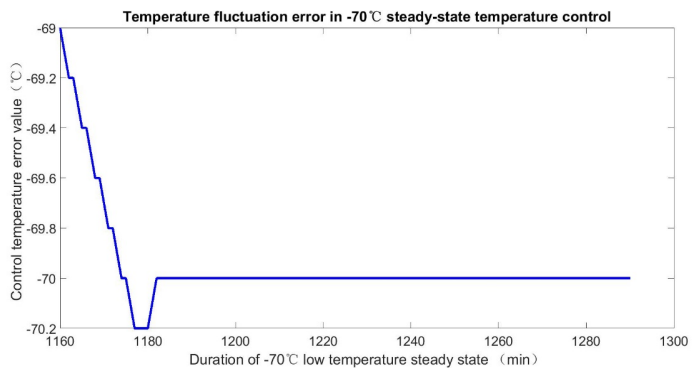

Figure 12. Temperature fluctuation error in $-70^{\circ} \mathrm{C}$ steady-state temperature control

\section{Conclusion}

A set of temperature control system for thermal vacuum test was successfully designed and developed based on the Modbus RTU serial communication protocol on the LabVIEW platform. The master-slave serial communication method ensures the reliability of information transmission and improves the communication transmission efficiency ${ }^{[8]}$. The temperature control system has been tested in the thermal vacuum test, which has achieved the expected goal and has practical application value.

\section{References}

1. HUANG B C, MA Y L. Spacecraft space environment test technology[M]. National Defense Industry Press. 2002 :84-91.

2. SUN Y, LIU G T, GU Z F, ZHAN H Y. Self-tuning PID control strategy for thermal vacuum tests[J]. Spacecraft Environment Engineering. 2016, 33(3) : 333-336.

3. Wright M. Multi-computer VXIbus system set up and use[J]. Aerospace and Electronic System Magazine, IEEE. 1998, 13(9) :17-21.

4. LI N, XU J, YANG L H. A new technique for automatic temperature control of cryogenic blackbody[J]. Spacecraft Environment Engineering. 2011, 28(4):362-366.

5. Claudio Urrea, Claudio Morales, John Kern. Implementation of error detection and correction in the Modbus-RTU serial protocol[J]. International Journal of Critical Infrastructure Protection. 2016, 15(1) :27-37.

6. Ramabadran T V, Gaitonde S S. A tutorial on CRC computations[J]. IEEE Micro. 1988, 8(4):62-75. 
7. Pradhan D, Lakshminarayanan L, Patil V. A LabVIEW based power analyzer[C]. Advances in Energy Converision Technologies(ICAECT).2014 International Conference :67-71.

8. KUANG Y C. Communication between PLC and Arduino based on Modbus protocol. Instrumentation and Measurement, Computer, Communication and Control (IMCCC), 2014:370-373. 